\title{
Commentary
}

\section{Status of semen analysis - comment on "A survey on the status of semen analysis in 118 laboratories in China" by Jin-Chun Lu et al. in Asian Journal of Andrology}

\author{
Hugh William Gordon Baker
}

Department of Obstetrics and Gynaecology, University of Melbourne and Melbourne IVF Reproductive Services, The Royal Women's Hospital, Parkville 3052 Victoria, Australia

Fax:+61-3-9347-1761_E-mail:g.baker@unimelb.edu.au

Asian Journal of Andrology (2010) 12: 115-117. doi: 10.1038/aja.2009.7

The paper by Lu et al. [1] "A survey on the status of semen analysis in 118 laboratories in China" provides a major review of the current methods of semen analysis in mainland China. It involved a 36-item questionnaire completed by technicians in 118 of a possible 145 potential semen laboratories. Recruitment for the study was via contacts developed at Chinese Andrology meetings and training sessions between 2005 and 2007. It is not stated how comprehensive this strategy would be for accessing all semen laboratories in China. About half the participating laboratories were in general hospitals and the others in hospitals with academic affiliations or family planning institutes. Some who did not complete the questionnaire were found to be not involved in semen analysis or their institution did not have a semen laboratory and thus the impressive response rate is probably even higher-a notable characteristic of Chinese medicine! The results led the authors to call for better standardization of semen analysis.

My comments and suggestions are made from the point of view of semen analysis used for assessment and management of infertility [2].

A number of shortcomings in the approaches used and the tests applied are apparent in the report of the survey. It is remarkable that:

1. about 50 percent of the laboratories used computer-aided semen analysis (CASA),

2. many recognized that semen analysis as currently performed was less than satisfactory,

3. ELISA was often used for antisperm antibodies,

4. semen biochemistry was performed routinely by 27 percent of laboratories,

5. quality assurance activities were absent and

6. methods not consistent with the 1999 World Health Organization (WHO) Semen Analysis manual [3] were probably used frequently.

It is widely accepted by laboratory accrediting bodies that methods such as those in the WHO manual should be adopted as the standard and that those in charge of laboratories that do not use these methods must justify their own variation and show it produces equivalent or superior results. WHO manual contains standard methods and acceptable variations in procedures and also gives other methods for optional tests and research tests. I will mainly confine my comments to the standard methods. I believe robust methods are needed for measurement of semen volume, sperm concentration, progressive and total motility, sperm morphology and sperm antibodies. Some aspects of semen assessment can be trimmed for clinical purposes for example viscosity, liquefaction, sperm vitality and semen biochemistry. Mere noting of extreme viscosity or defective liquefaction is sufficient for the clinician to be alerted to the fact that the semen analysis results may be invalid because of the abnormality of semen consistency. While $\mathrm{pH}$ could be done routinely it is really only of interest for patients with azoospermia or low semen volume in which case an acid $\mathrm{pH}$ may suggest the seminal vesicle contribution to the semen is missing. This assists in the diagnosis of bilateral congenital absence of the vasa and ejaculatory duct obstruction. I believe this is sufficient for clinical purposes and that other semen biochemistry does not help although fructose could be measured as an alternative to $\mathrm{pH}$ to indicate missing seminal vesicle fluid. Sperm vitality can be assessed 
routinely as a check on sperm motility; both should give similar results. However for clinical purposes vitality is only of interest if sperm motility is low as it would assist in the diagnosis of necrozoospermia [2].

It is surprising that none of the laboratories assess semen volume by weighing which is the simplest approach in the WHO manual [3] "2.3.3 Volume The volume of the ejaculate may be measured... or by weighing standard containers with and without semen." If standard capped collection cups are used the variation in weight between cups is small and weighing the cup with semen and subtracting the average weight of several empty cups is more accurate than tipping the semen into a graduated cylinder because of the variable loss of the semen from it sticking to the wall of the cup [4].

Sperm antibodies should be measured on one sample of an infertile man as sperm autoimmunity causes about 5 percent of male infertility and there is no characteristic appearance in the semen analysis. Sperm agglutination is not a good indicator of sperm autoimmunity. If the presence of sperm antibodies is not discovered it is possible the patient would be treated by standard IVF and have failure of fertilization because of the undetected sperm autoimmunity. Therefore a sperm antibody test is recommended in the WHO manual [3] as a standard procedure. A sperm cervical mucus penetration test would be an alternative effective screening test for sperm autoimmunity. It is of critical importance that the sperm antibody test used is one of those that are established to detect pathological sperm antibodies reliably. These are the immunobead test (IBT) and the sperm immobilization test which were only used in under 10 percent of the Chinese laboratories. Over $50 \%$ of laboratories offering sperm antibody testing use ELISA which provides results that are unrelated to those of the IBT and would be highly misleading clinically [5].

The survey found greatest dissatisfaction with sperm morphology assessment. Sperm morphology assessed at low magnification without staining the sperm is unlikely to be useful. Quality assurance for sperm morphology on fixed and stained smears is particularly poor with often no agreement between results of different laboratories [6-9]. This is important because persistently poor sperm morphology in several samples is a very good indicator of a sperm defect and the need for intracytoplasmic sperm injection (ICSI) instead of standard IVF to avoid a zero or low fertilization rate. Introduction of fully automated methods should overcome the differences between laboratories for sperm morphology assessments [10]. In the meantime the clinicians need to know how to interpret the results from the specific laboratories they use.

The high usage of CASA in China is notable with over 50 percent of the laboratories using it. About $30 \%$ of these laboratories use CASA on its own but most use combined manual and CASA methods. It is possible to get excellent results for sperm concentration and motility with CASA but there are many traps [10]. Many systems still do not distinguish debris from sperm and there can be large sperm concentration-dependent biases. External quality assurance is critical for demonstrating the adequacy of the various CASA systems being used. CASA is also used for sperm morphology by about 25 percent of laboratories and these semiautomated methods are unlikely to be accurate.

Although the usefulness of quality assurance in semen laboratories has been questioned and there are few examples where it has revealed correctable problems there is a strong belief that it should be routine in semen analysis as in other areas of clinical testing [11-13]. Quality assurance activities are critical for assessing the accuracy and precision of the laboratory. Studies performed during training of technicians and quality assurance procedures show that the variability of results is very high. While with training coefficients of variation for results of sperm concentration and motility done on the same samples by different people can be brought down to about $15 \%$, greater variability is found before training even with technicians regularly performing semen analysis [14-17].

The fact that no quality assurance is conducted, that nonstandard methods are employed and that there are variations in the ranges of tests offered is not ideal but, to be fair this is not unique to China, previous surveys have shown considerable areas for improvement whereever they have been conducted [13, 18-21]. I suspect these problems are inherent in manual methods of semen analysis and will persist until new techniques of male fertility assessment are developed. Dr Lu and his colleagues are to be commended for their efforts to improve semen analysis in China [22].

\section{References}

1 Lu JC, Zhang HY, Hu YA, Huang YF, Lü NQ. A survey on the status of semen analysis in 118 laboratories in China. Asian J Androl 2010; 11: 104-10.

2 Baker HW. Clinical management of male infertility. Available from http://www.endotext.org. DeGroot LJ. Editor. 2009.

3 World Health Organization. WHO Laboratory Manual for the Examination of Human Semen and Sperm-Cervical Mucus Interaction, 4th edn. Cambridge: Cambridge University Press; 1999.

4 Brazil C, Swan SH, Drobnis EZ, Liu F, Wang C, et al. Standardized methods for semen evaluation in a multicenter research study. J Androl 2004; 25: 635-44.

5 Clarke G, Baker HW. Immunological evaluation of male infertility,. In: Male reproductive function pathophysiology and treatment. Kan- 
deel F, Editor. New York, Informa. 2007; p293-300.

6 Matson PL. External quality assessment for semen analysis and sperm antibody detection: results of a pilot scheme. Hum Reprod 1995; 10: $620-5$.

7 Ombelet W, Bosmans E, Janssen M, Cox A, Maes M, et al. Multicenter study on reproducibility of sperm morphology assessments. Arch Androl 1998; 41: 103-14.

8 McLachlan RI, Baker HWG, Clarke GN, Harrison KL, Matson PL, et al. Semen analysis: its place in modern reproductive medical practice. Pathology 2003; 35: 25-33.

9 Castilla JA, Morancho-Zaragoza J, Aguilar J, Prats-Gimenez R, Gonzalvo MC, et al. Quality specifications for seminal parameters based on the state of the art. Hum Reprod 2005; 20: 2573-8.

10 Garrett C, Liu DY, Clarke GN, Rushford DD, Baker HW. Automated semen analysis: 'zona pellucida preferred' sperm morphometry and straight-line velocity are related to pregnancy rate in subfertile couples. Hum Reprod 2003; 18: 1643-9.

11 Jequier AM. Is quality assurance in semen analysis still really necessary? A clinician's viewpoint. Hum Reprod 2005; 20: $2039-42$.

12 Knuth UA, Neuwinger J, Nieschlag E. Bias to routine semen analysis by uncontrolled changes in laboratory environment--detection by long-term sampling of monthly means for quality control. Int J Androl 1989; 12: 375-83.

13 Pacey AA. Is quality assurance in semen analysis still really necessary? A view from the andrology laboratory. Hum Reprod 2006; 21: 1105-9.

14 Jequier AM, Ukombe EB. Errors inherent in the performance of a routine semen analysis. Br J Urol 1983; 55: 434-6.

15 Auger J, Eustache F, Ducot B, Blandin T, Daudin M, et al. Intra- and inter-individual variability in human sperm concentration, motility and vitality assessment during a workshop involving ten laboratories. Hum Reprod 2000; 15: 2360-8.

16 Brazil C, Swan SH, Tollner CR, Treece C, Drobnis EZ, et al. Quality control of laboratory methods for semen evaluation in a multicenter research study. J Androl 2004; 25: 645-56.

17 Toft G, Rignell-Hydbom A, Tyrkiel E, Shvets M, Giwercman A. Quality control workshops in standardization of sperm concentration and motility assessment in multicentre studies. Int J Androl 2005; 28: 144-9.

18 Souter VL, Irvine DS, Templeton AA. Laboratory techniques for semen analysis: a Scottish survey. Health Bull (Edinb) 1997; 55: 140-9.

19 Keel BA, Quinn P, Schmidt CF Jr., Serafy NT Jr., Serafy NT Sr., et al. Results of the American Association of Bioanalysts national proficiency testing programme in andrology. Hum Reprod 2000; 15: 680-6.

20 Keel BA. How reliable are results from the semen analysis? Fertil Steril 2004; 82: 41-4.

21 Riddell D, Pacey A, Whittington K. Lack of compliance by UK andrology laboratories with World Health Organization recommendations for sperm morphology assessment. Hum Reprod 2005; 20: 3441-5.

22 Huang YF, Lu JC. [Advances in standardization and quality control for the analysis of sperm quality parameters]. Zhonghua Nan Ke Xue 2007; 13: 963-8. 